\title{
Cap au Nord: de nouvelles routes pour le soja brésilien
}

PAR

\section{Martine GUIBERT}

GRAL-CNRS

La restructuration actuelle de la logistique de transport au Brésil participe de l'objectif que se sont donné les acteurs des filières agroindustrielles : diminuer le custo Brasil ou surcoût lié, entre autres ${ }^{1}$, à la désorganisation et à la vétusté des différents modes.d'acheminement terrestre. C'est un enjeu considérable car, dans ce pays-continent, de grandes distances séparent les zones de production et les foyers urbains de consommation, localisés, pour la plupart, le long des côtes. Alors que la concurrence s'exacerbe sur les marchés internationaux, le Brésil doit avoir les moyens, grâce à l'efficience de ses ports d'expédition, de rester l'un des principaux exportateurs de produits agricoles bruts et transformés.

\footnotetext{
1 Les Brésiliens désignent par custo Brasil un ensemble de facteurs négatifs qui surenchérissent les coûts de production et de commercialisation des produits agricoles et industriels : politique macro-économique, politique fiscale, mauvais fonctionnement de la logistique, du stockage à la livraison chez le client ou aux opérations de débarquement et embarquement portuaires.
} 
Plusieurs projets novateurs de couloirs de transport multimodaux ${ }^{2}$ sont en cours de concrétisation. Certains accompagnent la mise en culture actuelle, basée sur le soja, de vastes régions du Nord-Ouest et du Centre-Ouest. D'autres doivent modifier les circuits utilisés dans les régions traditionnellement cultivées. Celui de la voie fluviale ParaguayParaná consiste à développer un axe de transport intégré entre les pays du Mercosur.

Dans ce travail, nous nous intéressons spécifiquement au cas du couloir de transport multimodal du Nord-Ouest. Mis en service en avril 1997, il se structure autour du Rio Madeira et de l'Amazone, et dessert la Chapada dos Parecis, grande zone de développement du soja, au cœur de l'Etat du Mato Grosso. La finalité est l'expédition de graines de soja, du port d'Itacoatiara (sur l'Amazone) jusqu'au port européen de Rotterdam et jusqu'aux ports asiatiques. Les coûts et les délais sont moins élevés que ceux des ports du Sud du pays (Santos dans la banlieue de São Paulo, Paranaguá près de Curitiba), plus éloignés des destinations finales et plus désorganisés.

En préliminaire, nous évoquons l'expansion spatiale de la culture du soja jusqu'aux abords de l'Amazonie et nous présentons succinctement les autres projets de couloirs de transport multimodaux.

\section{Le soja, support de la dernière frontière agricole}

Avec le maïs, les haricots, le riz, la canne à sucre, le café et les agrumes, le soja figure parmi les principales productions agricoles du Brésil. Sur un volume annuel total variant entre 70 et 80 millions de tonnes, obtenues sur 50 millions d'hectares, le soja représente entre 25 et 26 millions de tonnes, récoltées sur 11 à 12 millions d'hectares (rendement moyen d'environ 2,3 tonnes par hectare). Les conditions climatiques favorables au moment des semis, le dynamisme du marché brésilien et les prix internationaux élevés ont stimulé la bonne récolte de

2 Nous choisissons de traduire l'expression brésilienne "corredor de transporte multimodal" par "couloir de transport multimodal". Elle désigne un système de transport structuré autour d'un axe (un fleuve) qui combine au minimum deux modes de transport continentaux différents. Dans la littérature française spécialisée, nous retrouvons cette expression, ainsi que celle de "système de transport multimodal" ou celle de "couloir de transport combiné" (voir Bourdillon, 1991). 
la campagne 1996/973. Les dernières estimations (USDA - Ministère de l'Agriculture des Etats-Unis) avancent une production de 77,4 millions de tonnes de grains (céréales et oléagineux). 26,4 millions de tonnes de soja seraient récoltées sur 11,4 millions d'hectares. Ce chiffre correspond à près de $20 \%$ dè la production mondiale (132,5 millions de tonnes), les Etats-Unis fournissant environ $50 \%$ et l'Argentine plus de $9 \%$.

C'est dans les années 1970 que le Brésil est devenu le second producteur mondial de soja. Sa culture s'est d'abord développée au Sud et au Sud-Est et s'est vite étendue à la région des Cerrados, dans les Etats du Mato Grosso do Sul, Goiás et Minas Gerais. Elle a ensuite motivé la remontée vers le Nord de producteurs à la recherche de terres bon marché. Depuis quelques années, elle s'est installée plus vers le CentreOuest, dans l'Etat du Mato Grosso et, dernièrement, dans le CentreNord, au confins des Etats du Maranhão, de Bahia, du Tocantins et du Piauí.

Aujourd'hui, les Etats du Paraná, du Rio Grande do Sul et du Mato Grosso restent les principaux producteurs; ils proposent près des $2 / 3 \mathrm{du}$ soja récolté.

\footnotetext{
${ }^{3}$ Cette conjoncture commerciale positive a été amplifiée par la fin de l'ICMS - Imposto de Circulação de Mercadorias e Serviços - sur les graines de soja. Elle devrait permettre aux producteurs d'assainir leur situation financière (fonds disponibles pour diminuer l'endettement) et de réaliser des investissements en matériel, semences sélectionnées, engrais et produits phytosanitaires. Cette embellie est très appréciée, après des années de crise de la rente agricole qui a condamné des milliers de producteurs à vendre leurs terres.
} 
Tableau $1:$ La distribution spatiale de la culture du soja

\begin{tabular}{|c|c|c|c|c|c|c|c|c|}
\hline \multirow[b]{2}{*}{ Localisation } & \multicolumn{4}{|c|}{ Superficie } & \multicolumn{4}{|c|}{ Production } \\
\hline & $1993 / 94$ & $1994 / 95$ & $1995 / 96$ & $\begin{array}{c}\% \\
95 / 96 \\
\end{array}$ & $1993 / 94$ & $1994 / 95$ & $1995 / 96$ & $\begin{array}{c}\% \\
25 / 96 \\
\end{array}$ \\
\hline \multicolumn{9}{|l|}{$\begin{array}{c}\text { Etats du } \\
\text { Sud et Sud- } \\
\text { Est }\end{array}$} \\
\hline Paraná & 2154,1 & 2125,0 & 2311,5 & 21,58 & 5332,9 & 5591,0 & 6009,9 & 25,04 \\
\hline $\begin{array}{c}\text { Rio Grande } \\
\text { do Sul }\end{array}$ & 3185,1 & 3007,7 & 2864,3 & 26,74 & 5442,7 & 5886,2 & 4497,0 & 18,73 \\
\hline $\begin{array}{c}\text { Mato Grosso } \\
\text { do Sul }\end{array}$ & 1101,7 & 1042,7 & 878,3 & 8,20 & 2392,5 & 2358,9 & 2064,0 & 8,60 \\
\hline São Paulo & 553,9 & 540,0 & 563,6 & 5,26 & 1230,9 & 1165,0 & 1223,0 & 5,09 \\
\hline $\begin{array}{c}\text { Santa } \\
\text { Catarina } \\
\end{array}$ & 217,3 & 205,0 & 222,4 & 2,07 & 434,4 & 443,3 & 467,0 & 0,02 \\
\hline \multicolumn{9}{|l|}{$\begin{array}{c}\text { Etats du } \\
\text { Nord-Ouest }\end{array}$} \\
\hline $\begin{array}{l}\text { Mato } \\
\text { Grosso }\end{array}$ & 2023,0 & 2280,4 & 1859,3 & 17,36 & 5319,8 & 5685,5 & 4555,3 & 18,98 \\
\hline \multicolumn{9}{|l|}{$\begin{array}{c}\text { Etats du } \\
\text { Centre- } \\
\text { Ouest }\end{array}$} \\
\hline Goiás & 1110,5 & 1123,0 & 909,4 & 8,49 & 2331,0 & 2153,7 & 1955,2 & 8,14 \\
\hline Minas Gerais & 591,5 & 601,3 & 528,0 & 4,93 & 1268,9 & 1199,7 & 1082,4 & 4,51 \\
\hline $\begin{array}{l}\text { Etats du } \\
\text { Centre-Est }\end{array}$ & & & & . & & & & \\
\hline Bahia & 434,0 & 470,6 & 433,0 & 4,04 & 873,4 & 1072,9 & 909,3 & 3,79 \\
\hline Maranhăo & 62,9 & 87,5 & 85,3 & 0,01 & 140,6 & 169,4 & 191,9 & 0,01 \\
\hline $\begin{array}{l}\text { TOTAL } \\
\text { BRESIL }\end{array}$ & 11514,4 & 11545,4 & 10709,5 & 100 & 24912,4 & 25843,0 & $24000^{*}$ & 100 \\
\hline
\end{tabular}

Superficie en millions d'hectares. Production en millions de tonnes. ${ }^{*}$ : estimation Source : Elaboration personnelle à partir de Vieira, 1997 (données de base : CONAB)

Les deux foyers d'expansion récents de la Chapada dos Parecis et de la région Balsas-Barreiras délimitent, chacun à l'une de ses deux extrémités, la dernière frontière agricole ouverte au centre du territoire brésilien, en bordure du bassin amazonien. 
Avec $165000 \mathrm{~km}^{2}$ et environ 200000 habitants, la région de la Chapada dos Parecis se situe dans l'Etat du Mato Grosso. De par sa topographie plane, ses sols profonds et rouges, son climat ensoleille et régulièrement pluvieux, elle constitue l'un des réservoirs de terres les plus potentiellement productives du Brésil. En 1994, sur 1,5 million d'hectares valorisés, le soja occupait plus de 1,1 million d'hectares. Avec un rendement de 2,67 tonnes par hectare, plus élevé que la moyenne nationale, la production a frôlé les 3 millions de tonnes (Ministerio dos Transportes, 1996 : 4). En 1997, 5 millions de tonnes de soja devraient être récoltées au total dans l'Etat du Mato Grosso (Folha de São Paulo, 15 mars 1997). Le groupe fondé par André Maggi est l'un des principaux maîtres d'oeuvre de la mise en culture de cette zone. Après la consolidation de ses activités à l'Ouest de l'Etat du Paraná et près de Cuiabá, à l'Ouest de l'Etat du Mato Grosso, il a étendu ses propriétés au Nord de l'Etat du Mato Grosso. La nouvelle ville de Sapezal (4 000 habitants) lui doit son existence, ses infrastructures, ses projets de développement et sa transformation récente en município. Considéré comme "o rei da soja", le groupe projette l'achat de nouvelles terres encore plus au Nord, à Humaitá, au Sud de l'Etat d'Amazonas. Il possèderait $95 \%$ des 40000 hectares qu'il consacre à cette culture. Il produira, en 1997, autour de 100000 tonnes. Avec 600 à 700000 tonnes vendues, il est l'un des principaux négociants et, surtout, il aspire à devenir l'un des plus importants exportateurs du Brésil. La mise en service du couloir de transport multimodal Nord-Ouest, qu'il soutient très activement, devrait lui permettre d'expédier, en 1997, 420000 tonnes (Folba de São Paulo, 26 janvier 1997).

Le second foyer se compose de la région de Balsas, au sud de l'Etat du Maranhão, et de celle de Barreiras, au sud de l'Etat du Piauí. Dans la région de Balsas, l'arrivée quotidienne d'agriculteurs, venus du Sud pour acheter des terres éventuellement défrichées et encore peu chères, provoque le réveil et la création de petites villes, la constitution de fazendas et une croissance très forte des productions agricoles : 8000 tonnes de grains récoltées en 1990, 254000 en 1997, 500000 prévues en 2000. Dans la région de Barreiras, l'expansion du soja se fait dans le cadre du programme PRODECER - PROgrama de DEsenvolvimento do CERrado-, financé à $60 \%$ par le Japon. "O interesse do governo japonês nesses financiamentos é aumentar a oferta mundial de alimentos, para não ficar dependente de poucos fornecedores" (Veja, 2 avril 1997). La phase actuelle prévoit l'implantation de colonies agricoles sur 40000 
hectares. En 1996, 20 à 25000 hectares ont été semés et 23000 tonnes récoltées (Folha de São Paulo, 23 octobre 1996).

Gravitant autour de ces deux zones pionnières, d'immenses superficies devraient être progressivement mises en valeur : Sud de l'Etat d'Amazonas, vers Humaitá, Nord-Est de l'Etat d'Acre, vers Rio Branco, terres le long du Rio Madeira et de l'Araguaia, Ouest de l'Etat de Bahia, etc. Autrement dit, de Rio Branco à Barreiras, "segundo o Ministério da Agricultura, existem 45 milhōes de hectares de terreno cultivável nessa faixa, ainda inexplorados. [...] Esses números refletem o tamanho da potencialidade econômica dessa faixa do Brasil Central" (Veja, 2 avril 1997). Seule la mise en culture des 16 millions d'hectares de la Chapada dos Parecis permettrait d'obtenir, après amélioration des rendements, l'équivalent de 60 millions de tonnes de soja (soit la production des Etats-Unis ou plus de deux fois la production brésilienne actuelle).

L'ouverture de fronts agricoles génère le développement d'activités industrielles et de services. Etant donné sa forte consommation de fertilisants, de pesticides et de semences sélectionnées, et ses besoins en technologie avancée, la production du soja s'avère très liée au secteur agro-industriel d'amont: Elle s'intègre néanmoins encore plus à l'agroindustrie d'aval car, comme les autres oléo-protéagineux (tournesol, colza, arachide, coton), c'est une production destinée à être transforméét.

En moyenne, au Brésil, $85 \%$ de la récolte est triturée. En 1996, plus de 20 millions de tonnes de graines ont ainsi été industrialisées pour obtenir 15,3 millions de tonnes de tourteaux et près de 3,7 millions de tonnes d'huile. Afin de limiter le fret, les unités de transformation du soja se sont multipliées dans les régions de production et près des points de consommation ou d'expédition. Concentrées dans le Sud et Sud-Est, elles s'installent petit. à petit dans le Centre, suivant l'expansion géographique du soja ${ }^{5}$. En fait,

4 La trituration de la graine de soja permet d'obtenir de l'huile, propre à la consommation humaine après son raffinage, et des sous-produits à fort taux protéique, tels les tourteaux incorporés dans les rations des animaux élevés intensivement pour la production de viandes blanches (volailles, porcs) ou de lait (vaches laitières).

5 Par exemple, la firme multinationale nord-américaine Cargill serait sur le point d'acheter l'huilerie qu'elle loue à Barreiras. La société brésilienne Ceval a construit une unité de trituration de plus de 600000 tonnes par an à São Luis (Etat du Maranhảo). 
a marcha da indístria processadora para as regiōes de fronteira, seguindo o caminho da matéria-prima (e trazendo consigo outros segmentos da mesma indistria), pode ser explicada pelo elevado percentual (aproximadamente 65 \%) que representa a matéria-prima nos custos operacionais de uma planta processadora de obleos vegetais. Para alguns especialistas, a instalação de induistrias esmagadoras nas regiöes de fronteira só será plenamente viabilizada com a preseņ̧a de unidades de conversäo de proteina vegetal em animal (produçáo avicola, por exemplo). O frete do transporte de óleo $e$ farelo é muito elevado, o que justificaria deslocar um produto com maior valor agregado. (Burnquist, 1994 : 53)

En exportant $66 \%$ des tourteaux qu'il produit, $40 \%$ de l'huile brute et $25 \%$ des graines, le Brésil appartient au groupe de tête des exportateurs mondiaux de produits à base de soja. Ses deux concurrents directs sont les Etats-Unis et l'Argentine. Il est le premier fournisseur de tourteaux, le deuxième d'huile brute et le troisième de graines. Ces trois produits (sans compter les aliments plus élaborés) représentent environ $10 \%$ des recettes à l'exportation de l'Etat brésilien et un tiers de celles composées exclusivement de produits agricoles et agro-industriels qui ont rapporté, en 1997, 14,5 milliards de dollars. Avec une baisse des ventes de produits dérivés ( 9,3 millions de tonnes de tourteaux contre 10,7 en 1996 et 1 million de tonnes d'huile contre 1,3 en 1996) et une hausse très importante de celles de graines $(8,5$ millions de tonnes contre $3,3$ en 1996$)^{6}$, le complexe soja devrait rapporter plus de 5,4 milliards de dollars (plus $22 \%$ par rapport à 1996) (Veja, 26 mars 1997).

Les principaux acheteurs sont 'l'Union Européenne, les pays du Moyen-Orient et ceux d'Asie du Sud-Est. Or, le glissement spatial de la culture du soja vers l'Ouest et le Centre du territoire a cré un nouveau handicap en matière de logistique de transport interne.

$O$ alargamento da área de cultivo para o cerrado, ao mesmo tempo que permitiu uma produtividade comparável às mais altas obtidas no mundo, introduziu problemas de transporte e de produçāo, que ao mesmo tempo que tornam o custo de produção mato-grossense o mais alto do pais, aumenta a distância média entre as áreas produtoras brasileiras e os portos para 895

6 Elle est une conséquence directe de lá suppression des $13 \%$ d'ICMS sur les exportations de graines, les tourteaux continuant à être taxés à $11 \%$ et l'huile à $8 \%$. Les acteurs du complexe soja ont donc placé plus de graines sur les marchés tiers et en ont importé 2 millions de tonnes (contre 1 million de tonnes en 1996) pour approvisionner leurs huileries (Voir Gazeta Mercantil, 5 septembre 1996, et Folha de Säo Paulo, 2 septembre 1996) 
$\mathrm{km}$, estimados em 1990, contra os $6.62 \mathrm{~km}$ calculados em 1980. (Vieira, $1997: 122)$

Ainsi, les nouvelles zones de production sont plus éloignées des installations portuaires d'expédition du Sud et du Sud-Est. En revanche, elles sont plus proches des ports de destination. Par exemple, il y a 5000 $\mathrm{km}$ de moins entre le port de Ponto da Madeira, près de São Luis, et celui de Rotterdam, qu'entre le port de Santos, près de São Paulo, et ce même port européen. Cette situation inédite, replacée dans un contexte international très compétitif, rend vitale une amélioration des conditions d'acheminement de la production vers ces points d'expédition plus rentables.

\section{Plusieurs projets de couloirs de transport multimodaux}

Au cours des années 1970 et 1980, "uma tendência de desconcentração geográfica da atividade econômica" et "uma significativa expansão do comércio intra-regional"7 (Castro, 1995 : 5) ont accru les besoins en logistique de transport. Or, par manque de modernisation et d'organisation, les réseaux de transport brésiliens continuent, à l'approche du XXIe siècle, à ne pas offrir de bonnes conditions pour l'écoulement des marchandises, notamment des différentes productions agricoles, aux volumes conséquents mais à faible valeur ajoutée. Bien que privilégié, le transport routier reste inadéquat. Les conditions de circulation sont dangereuses sur la plupart des routes et de nombreux goulots d'étranglement congestionnent le trafic, surtout au plus fort des récoltes. Le réseau ferroviaire actuel, identique à celui des années 1950, est délaissé. Les voies fluviales comme celles qui avaient été aménagées sur le Tietê, le Sáo Francisco ou l'Araguaia sont peu utilisées. $67 \%$ des produits issus du complexe soja sont ainsi transportés par la route, $28 \%$ le sont par le rail et $5 \%$ par voie fluviale. Aux Etats-Unis, ces proportions sont respectivement de $16 \%, 23 \%$ et $61 \%$ (Abiove, 1996).

Face à la hausse des coûts (le transport routier coûte neuf fois plus cher que le transport fluvial et trois fois plus cher que le transport ferroviaire), une nouvelle vision du transport s'est imposée chez les acteurs des différentes filières agro-industrielles.

7 La région du Sud-Est a perdu de l'importance dans la formation du PIB national, au profit des régions du Nord, Nord-Est et Centre-Ouest. La région Sud a maintenu sa participation. 
$O$ conceito via de transporte deve ser substituido por eixos de transporte [...] o que nada mais é do que a combinação de uma ou mais modalidades, que torna mais econômica a transferência de um bem desde a sua origem até o cliente... (Pavan, 1994 : 55)

Des projets de'transport multimodaux ont été élaborés qui structurent des "couloirs" d'acheminement des marchandises autour d'axes qui peuvent être, par exemple, un fleuve. Moyens de maîtrise et de valorisation de l'espace brésilien, ces couloirs constituent des enjeux économiques, sociaux et environnementaux considérables. En effet,

os corredores de transporte multimodais integrados (rodovia, ferrovia, hidrovia, porto, aerovia) objetivam a integraçăo racional e competitiva entre as áreas de produção e os centros de consumo do país ou pontos para exportaçäolimportação. (Licio, 1995 : 3)

La création de ces couloirs de transport multimodaux, insérés au cœur de régions en plein boom agricole, figure parmi les actions multisectorielles à mener dans le cadre du plan pluri-annuel 1996-1999 qui préconise plus de décentralisation, de dérégulation et de partenariat privé-public ${ }^{8}$. Leur aménagement a été chiffré à 800 millions de dollars.

Parmi les quatre projets de couloirs directement liés à l'écoulement des productions du complexe soja ${ }^{9}$, ceux du Centre-Nord et du CentreEst auront des impacts nationaux et ceux du Paraná-Paraguay et du Nord-Ouest auront des impacts continentaux.

\section{Le couloir Centre-Nord}

Il englobe l'Etat du Tocantins et s'étend du Nord-Ouest de l'Etat du Goiás au Sud de l'Etat du Maranhão, du Sud-Est de l'Etat du Pará et de l'Est de l'Etat du Mato Grosso à l'Etat du Piauí. 80 millions d'hectares

8 Par ailleurs, après la loi sur les ports de 1993 qui autorise la privatisation des installations portuaires fédérales, un programme concret d'investissements privés dans les ports d'expédition de produits agricoles et agro-industriels a été lancé en octobre 1995 (voir Folha de Säo Paulo, 6 janvier 1997 et 11 mai 1997). Un certain retard a été pris par rapport aux autres grands pays agro-exportateurs comme les Etats-Unis, organisés depuis longtemps, ou l'Argentine, qui a déjà restructuré et modernisé ses ports d'exportation et qui a des coûts d'embarquement 3 ou 4 fois inférieurs à ceux du Brésil.

${ }^{9}$ Il en existe quatre autres : le projer de couloir Nord (sortie maritime par la Caräbe), le projet de couloir Cuiabá-Santarém, le projet de couloir Nord-Est (axé sur le Rio São Francisco) et le projet Mercosul qui doit intégrer les réseaux de transport routiers, fluviaux et portuaires des quatre pays. 
sont concernés, dont plus de 21 millions cultivables. En 1993, 2,3 millions de tonnes de grains (riz, maïs, soja) ont été récoltées sur 3,8 millions d'hectares (Ministère des Transports, $1996: 10$ ). Les rendements déjà élevés des principaux noyaux de développement (région de Balsas et région de Barreiras) et l'avancée rapide de l'exécution du programme PRODECER laissent augurer de volumes de production agricoles futurs importants. La région présente aussi de bonnes aptitudes pour la sylviculture (production de cellulose à Imperatriz).

Ce couloir se scinde en deux parcours axés sur le Rio Araguaia et le Rio Tocantins, dont le dragage et le balisage constituent les principaux investissements. Le premier trajet emprunte le Rio das Mortes et le Rio Araguaia, de Nova Xavantina jusqu'à Xambioá, soit $1800 \mathrm{~km}$ de transport fluvial. Il se poursuit par la route jusqu'à Imperatriz ou Estreito. Les derniers $2100 \mathrm{~km}$ se font en chemin de fer, d'abord jusqu'à Açailândia et, enfin, jusqu'au port de Ponto da Madeira (São Luis) ou celui d'Itaqui, par la ferrovia Carajás $^{10}$. Le second trajet combine deux modes de transport : la voie fluviale de Miracema à Estreito $(480 \mathrm{~km}$ sur le Rio Tocantins), la voie ferrée d'Estreito à Imperatriz sur un tronçon Nord-Sud de $230 \mathrm{~km}$ (dont 120 en construction) et de Carajás au port de São Luis (Ponto da Madeira) ou Itaqui $(600 \mathrm{~km})$.

Le premier parcours a permis, en 1997, d'écouler la production de soja de la région de Balsas. Après un transport en camion jusqu'à la station ferroviaire d'Imperatriz, le soja est mis dans des wagons et emporté par la ferrovia Carajás. Il est ensuite chargé sur les bateaux amarrés dans. les ports maritimes concernés, en transitant ou non dans des silos de stockage. La diminution du fret est nette : "o frete de Balsas até Roterdã fica em 37 dólares a tonelada. O produtor paranense paga 41 reais para que sua soja chegue a Roterdā" (Veja, 12 avril 1997). Le second parcours est utilisé depuis cette même année, après la mise en service de $110 \mathrm{~km}$ de la voie ferrée Nord-Sud et sa combinaison avec la ferrovia Carajás. Les acteurs mettent en avant l'association d'une voie ferrée neuve et d'un port moderne et organisé, gérés par des entreprises privées et garantissant des produits à bas prix et compétitifs sur le marché mondial (Manchete Rurah avril 1996).

10 La Companhia Vale do Rio Doce gère la voie ferrée du Carajás qui transporte annuellement 49 millions de tonnes de marchandises, dont 45 millions de tonnes de minerai de fer (sa principale production), et 4 millions de tonnes de grains, dont 200000 tonnes de graines de soja. 
Plus généralement, le coût actuel du fret entre Nova Xavanitina et Rotterdam, après utilisation de la route et via Santos, le port du Sud, s'élève en moyenne à 87 dollars par tonne. L'utilisation du couloir Centre-Nord sur sa partie la plus longue (voie fluviale Nova Xavantina Xambioá sur P'Araguaia, route Xambioá - Imperatriz ou Estreito, voie ferrée Imperatriz - São Luis, voie maritime São Luis - Rotterdam) impliquerait des frais d'un montant de 71 dollars. par tonne. Après les premiers aménagements, ils seraient de 55 dollars par tonne, et de 37 dollars à partir de Miracema.

L'expansion du soja dans la zone d'influence du Rio Araguaia et l'intégration de celle-ci dans la zone d'influence du couloir Centre-Nord expliquent la décision de la Ceval de construire une unité de trituration dans l'Etat du Maranhão. "Com uma produção avaliada em um milhão de toneladas de grãos de soja, o Araguaia vai dar uma vantagem no volume transportado pelo corredor e garantir produção suficiente para o esmagamento' no Maranhão" (Gazeta Mercantil, 25 juillet 1996). L'entreprise de navigation Navbel doit fournir à la Ceval 4 barges de 2 500 tonnes chacune et un remorqueur. Par ailleurs, il existe les projets d'implantation, dans le municipe d'Imperatriz, d'une industrie de cellulose (420000 tonnes par an) et d'une industrie de fertilisants (50000 tonnes par an).

\section{Le couloir Centre-Est}

Il doit relier au système portuaire de Vitória-Tubarāo, dans l'Etat d'Espirito Santo, toute la région centrée sur le Nord-Ouest de l'Etat du Minas Gerais (Triângulo Mineiro) et la région du district fédéral de Brasilia. Environ 13 millions d'hectares seraient susceptibles d'être mis en valeur, dont près de 10 sont cultivables. Le chemin de fer est l'ossature de cet axe de transport destiné à acheminer, pour l'exportation, des produits agricoles, des produits sidérurgiques, de la cellulose et du minerai de fer et, pour l'importation, des fertilisants, du blé, des produits industriels (voitures). Appartenant à la Companhia Vale do Rio Doce, la voie ferrée entre le port de Tubarăo et la ville de Belo Horizonte fonctionne bien. Le tronçon suivant, entre Belo Horizonte et Pirapora, est en cours de privatisation. Son extension jusqu'à Unal devrait être construite par la compagnie privée. Les travaux de restructuration portuaire comprennent notamment la construction, à Tubarão, d'un terminal d'exportation exclusivement réservé à l'embarquement de 
graines et d'huile de soja. Les coûts de transport ferroviaire de produits agricoles d'Unaí à Tubaráo seraient de l'ordre de 38 dollars par tonne, contre 62 à l'heure actuelle d'Unaí à Santos.

\section{La voie fluviale Paraguay-Paraná}

Elle doit parcourir $3600 \mathrm{~km}$ de la ville de Cáceres (Etat du Mato Grosso) à l'embouchure du Río de la Plata. Au Brésil, elle concerne les Etats de São Paulo, du Paraná et du Mato Grosso do Sul. Son aménagement reste encore à l'étude tant les travaux sont importants et l'impact environnemental sérieux. Les parties déjà utilisées pour le transport de soja se situent dans la seconde partie de la future voie, notamment à partir d'Asunción (graines de soja produites au Paraguay et transportées pour trituration jusqu'aux unités argentines du port de Rosario). Début 1997, des travaux de dragage ont été entrepris à partir du port de San Martín (qui appartient au complexe portuaire argentin Rosario - San Lorenzo - San Martín). Ils ont porté à 32 pieds la profondeur du Río Paraná, ce qui permet le passage de bateaux de haute mer. Le second projet en cours d'approbation est celui de la construction d'un canal qui relierait le Río Paraguay et le Río Paraná. Il démarrerait au-dessus du gigantesque barrage hydroélectrique d'Itaipú, l'éviterait et se poursuivrait jusqu'au-dessus d'Asunción, la capitale paraguayenne. La connexion Tietê - Paraguay - Paraná serait alors possible.

\section{Le couloir Nord-Ouest}

Bien qu'inachevé, il est opérationnel sur sa majeure partie et a commencé à être utilisé en avril 1997. Une analyse plus précise de son organisation et des objectifs est motivée par son caractère prioritaire dans la phase actuelle d'aménagement du centre du territoire brésilien. Il doit irriguer les Etats du Mato Grosso, de Rondônia et d'Amazonas et les mettre en relation avec les pays andins limitrophes.

\section{Le couloir de transport multimodal : structure et enjeux ${ }^{11}$}

L'aire d'influence du couloir Nord-Ouest concerne 20 millions d'hectares potentiellement cultivables. Elle couvre, avant tout, la région

11 Les informations présentées ici sont extraites du document technique Corredor de transporte multimodal noroeste, établi et diffusé en juin 1996 par le Ministère des Transports brésilien. 
de la Chapada dos Parecis, dont l'épicentre est à $500 \mathrm{~km}$ au Nord-Ouest de Cuiabá. Elle dessert également les zones de Cerrados qui bordent, de part et d'autre, sur $150 \mathrm{~km}$, la route BR-364 (de Cuiabá à Rio Branco en passant par Porto Velho), les terres qui s'étendent le long du Rio Madeira (entre Porto Velho et Itacoatiara), le sud de l'Etat d'Amazonas (d'Humaitá à Apuí) et le Nord-Est de l'Etat d'Acre.

Le couloir combine la route, des zones de production à Porto Velho, la voie fluviale, sur le Rio Madeira, de Porto Velho à Itacoatiara (1 115 $\mathrm{km}$ ), et la voie maritime, sur l'Amazone, à partir d'Itacoatiara. Ainsii,

o projeto do Corredor de Transporte Multimodal Noroeste constituir-se-á em um eixo de macro-integraçāo para aquela regiāo do solo brasileiro [...]. A implantaçäo do aludido Corredor tem por intuito fomentar o escoamento da produçāo de gräos proveniente da "Nova Fronteira Agricola Brasileira", aproximadamente 4 milhöes ton / ano, situada a noroeste do Estado de Mato Grosso (regiäo da Chapada dos Parecis), por consequência, maximizando a competitividade desses produtos junto ao mercado internacional, face ao barateamento dos custos de transporte envolvidos. (Extrait d'un avis du Ministre Fédéral des Transports adressé au Ministre Fédéral de la Planification et du Budget)

Au début de 1996, sous le contrôle de l'Etat Fédéral brésilien, un protocole de partenariat a été signé entre les autorités des Etats d'Amazonas, du Mato Grosso et de Rondônia, les instances professionnelles impliquées et les responsables du groupe privé Maggi. Son objet était l'implantation du projet Transporte de Grãos, Granéis Sólidos e Insumos Agrícolas pela Hidrovia Madeira-Amazonas. Le groupe Maggi est l'instigateur du projet. Il détient $57 \%$ de la société mixte Hermasa Navegaçōes da Amazônia $S A$, créée avec le gouvernement de l'Etat d'Amazonas. Il finance à travers elle la plupart des travaux d'amélioration et de création des infrastructures fluviales et portuaires (Bolsa de Comercio de Rosario, 1997). Au total, 248 millions de dollars seront investis. 
Carte 1: Localisation et structure du couloir de transport multimodal Nord-Ouest
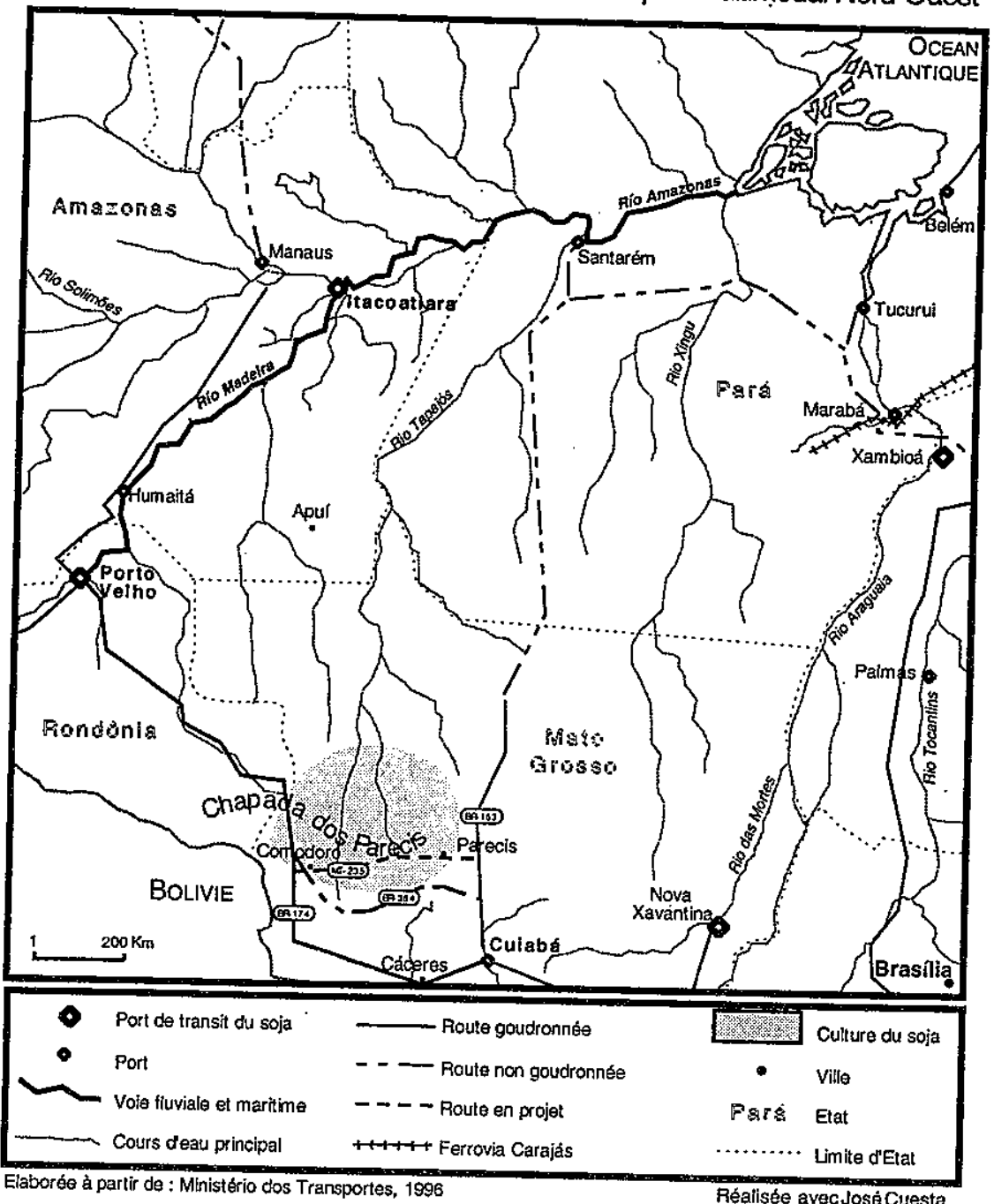
Le port de Porto Velho a été transformé pour recevoir les camions, stocker les grains (capacité de 50000 tonnes) et les embarquer (capacité de 800 tonnes par heure). Le groupe Maggi a apporte 5,5 millions de dollars et le gouvernement de l'Etat du Rondônia, 1,5 million. Le port d'Itacoatiara a été aménagé pour le stockage des grains (capacité de 90 000 tonnes) et leur transbordement des barges aux supertankers Panamax (capacité de chargement de 1500 tonnes par heure). Dans ce cas, le Gouvernement de l'Etat d'Amazonas et le groupe Maggi ont collaboré à hauteur respectivement de 12 et 16 millions de dollars, tandis qu'une banque nationale ${ }^{12}$ avançait 24 millions de dollars. L'entreprise Hermasa a acquis des barges et des remorqueurs pour 54 millions de dollars.

Parmi les autres grands travaux, il faut relever la réparation du tronçon routier Comodoro - Porto Velho et la construction de la route MT-235 (Rodovia Celeiro da Produção), sur $400 \mathrm{~km}$, entre Campo Novo dos Parecis et Comodoro. Elle raccordera la BR-163 et la BR-364 et traversera d'Est en Ouest la Chapada dos Parecis. Les 125 millions de dollars nécessaires seront issus de financements publics. Les 28 millions de dollars investis dans la cartographie, le balisage et le dragage du Rio Madeira le sont aussi. Il s'agit de rendre navigable le fleuve pendant toute l'année (contre 8 mois actuellement).

De acordo com o Departamento de Hidrovias Interiores, o calado minimo atualmente existente, de cerca de 2 metros, atende as necessidades iniciais do projeto HERMASA, podendo alcançar a cota de 3 metros, após a execufäo dos serviços de melhoramento na bidrovia, acompanbando os desdobramentos das fases de implantą̧äo do Projeto (1997 a 2002).

Actuellement, $90 \%$ du soja de la zone est écoulé par la route, vers le Sud, en direction du port de Paranaguá, près de Curitiba, ou du port de Santos, à $70 \mathrm{~km}$ de São Paulo. $2200 \mathrm{~km}$ sont parcourus en camion, sur des routes mal entretenues. A l'arrivée, les opérations de déchargement, de stockage et d'embarquement se passent dans de mauvaises conditions, du fait de la désorganisation générale et de la vétusté des installations portuaires. L'attente est toujours longue : il faut une semaine en moyenne pour embarquer le soja dans le port de Paranaguá et 12 jours

\footnotetext{
12 Il s'agit de la BNDES - Banco Nacional de Desenvolvimento Econômico e Social -. Elle fait crédit à des taux avantageux aux entreprises nationales désireuses de développer un projet d'industrialisation.
} 
dans celui de Santos. Il en résulte un surcoût qui n'existe pas avec le couloir de transport multimodal du Nord-Ouest .

Début avril 1997, 300000 tonnes de graines de soja (100 000 tonnes produites et 200000 tonnes achetées. par le groupe Maggi) ont inauguré le trajet. Transportées en camion sur la route BR-364, de la Chapada dos Parecis à Porto Velho, elles ont été chargées en vrac sur des convois composés d'un remorqueur et de six barges de 11600 tonnes chacune. Guidés par des ordinateurs qui analysent des informations satellitaires, les convois ont parcouru plus de $1000 \mathrm{~km}$ sur le Rio Madeira jusqu'au port d'Itacoatiara. Une semaine est nécessaire pour faire l'aller et retour. Les graines de soja ont été ensuite transbordées, soit directement, soit après stockage en silos, dans des navires de haute mer, type Panamax de 90000 tonnes, qui ont gagné l'Océan Atlantique, via l'Amazone ${ }^{13}$. En 1998, il est prévu d'écouler ainsi 600000 tonnes, et plus tard, 1 million de tonnes.

La comparaison des frais de transport entre le trajet classique Chapada-Santos-Rotterdam et le nouvel axe d'écoulement Chapada Porto Velho - Itacoatiara - Rotterdam est sans appel quant à l'importance de l'économie réalisée : dans le premier cas, ils s'élèvent à 102 dollars par tonne; dans le second, à 79 dollars par tonne. Avec l'augmentation des volumes, ils devraient encore baisser pour se stabiliser autour de 55 dollars par tonne (Tableau 2).

\footnotetext{
${ }^{13}$ Les acheteurs sont deux autres géants du soja : Cargill et le groupe Sumitomo.
} 
Tableau 2:

Le coût du fret Chapada dos Parecis - Santos - Rotterdam

\begin{tabular}{|l|r|r|}
\cline { 2 - 3 } \multicolumn{1}{c|}{} & \multicolumn{2}{c|}{ Coûts et prix en dollars par tonne } \\
\hline Trajet traditionnel & Aujourd'hui & \multicolumn{1}{c|}{ A long terme } \\
\hline Cotation Rotterdam 1996 & 293 & 293 \\
\hline $\begin{array}{rrr}\text { Chapada dos Parecis - Santos routier } \\
\text { fret maritime et prix FOB }\end{array}$ & 70 & \\
\hline $\begin{array}{l}\text { Santos - Rotcerdam } \\
\text { Impôts }\end{array}$ & 32 & 29 \\
\hline Gains & 43 & 0 \\
\hline
\end{tabular}

\begin{tabular}{|c|c|c|}
\hline & \multicolumn{2}{|c|}{ Coûts et prix en dollars par tonne } \\
\hline Nouveau trajet & Aujourd'hui & A long terme \\
\hline Cotation Rotterdam 1996 & 293 & 293 \\
\hline $\begin{array}{r}\text { Chapada dos Parecis - Porto Velho } \\
\text { fret routier } \\
\end{array}$ & 30 & 20 \\
\hline \begin{tabular}{|r|} 
Porto Velho - Itacoatiara \\
fret fluvial \\
prix FOB
\end{tabular} & $\begin{array}{r}19 \\
9\end{array}$ & $\begin{array}{r}13 \\
6\end{array}$ \\
\hline $\begin{array}{r}\text { Itacoatiara - Rotterdam } \\
\text { fret maritime } \\
\end{array}$ & 21 & 16 \\
\hline Impôts & 43 & 0 \\
\hline Gains & 171 & 248 \\
\hline
\end{tabular}

Source : Vieira, 1997

Un enjeui réside donc dans l'amélioration de la compétitivité du Brésil sur le marché mondial du soja et son renforcement vis-à-vis du premier exportateur mondial, les Etats-Unis : 


\begin{abstract}
... com essa hidrovia, a soja brasileira deverá chegar aos portos europeus a um custo inferior ao norte-americano - os maiores produtores. Nos EUA, o custo de produção da tonelada de soja vai de US\$130,00 a US\$160,00. No Brasil, o valor mais baixo (obtido na soja escoada pela bidrovia do Madeira) é de R\$113,00. (Folha de Säo Paulo, 12 avril 1997)
\end{abstract}

Un autre enjeu consiste à consolider la frontière agricole ouverte au Nord-Ouest en la rentabilisant et en introduisant des activités agroindustrielles, que ce soit dans les Etats du Rondônia ou d'Amazonas, ou dans des zones plus en retrait des Etats d'Acre, Pará et Roraima (Folha de Säo Paulo, 25 mars 1997).

A diversificaçâo de culturas, a implantação de agroindristrias, dentro de um modelo de desenvolvimento auto-sustentado, e o surgimento de novos negócios, säo possibilidades conseqüentes do novo ciclo. Configura-se iminente a instalaçäo dos seguintes projetos agroindustriais : açucar e álcool, sub-produtos do milho, proteinas animais (frigorifico e criatórios), óleos vegetais, fécula de mandioca e rafōos animais. (Ministère des Transports, 1996 : 19).

Le groupe Maggi a élaboré de tels projets dont il ne conçoit la réalisation qu’à partir du moment où l'infrastructure existe.

Dans une perspective géographique dépassant ces enjeux à l'échelle mondiale et ces objectifs de développement à l'échelle nationale, l'implantation du couloir de transport multimodal Nord-Ouest renvoie également à l'échelle régionale (sous-continentale). La proximité géographique de plusieurs pays sud-américains (Bolivie, Colombie, Pérou) permet de supposer qu'ils pourraient être des acheteurs importants des productions agricoles et agro-industrielles envisagées dans les zones traversées qui sont "...geograficamente e culturalmente mais próximos desses países do que dos estados brasileiros do Sul e Sudeste..." (MAARA, 1996:10). De même, dans le cadre de l'intégration régionale sud-américaine et avec comme objectif les marchés asiatiques, les activités d'exportation des produits brésiliens peuvent être envisagés à partir des ports du Pacifique (ports d'Esmeralda et de Paita en Equateur, port de Matarani au Pérou). Dès lors, la multimodalité supposerait de privilégier le transport ferroviaire et fluvial. La route, trop chère, serait un support et un moyen complémentaire. 


\section{Conclusion}

Le corridor de transport multimodal Nord-Ouest est un exemple de la nouvelle politique des autorités brésiliennes en matière d'acheminement des produits issus du complexe soja. L'amélioration de la compétitivité du Brésil sur le marché mondial est d'autant plus nécessaire que les perspectives d'exportation sont bonnes : réduction des subventions aux producteurs américains et européens concurrents, baisse globale de la production et hausse de la demande, faible disponibilité de nouvelles terres, consolidation des marchés européens, asiatiques et sudaméricains. Le défi consiste à adopter des politiques macro-économiques et agricoles adéquates et à développer des moyens logistiques efficaces.

L'aménagement de ports modernes est en cours sur les voies fluviales brésiliennes. Certains sont des ports dits de troisième génération, c'est-àdire des ports qui intégrent des activités de manutention et de commercialisation, et où est créée de la valeur ajoutée, par implantation d'industries. Les ports dits de deuxième génération, développés petit à petit dans les années 1980 , conçentraient seulement des centres de services pour le transport et le commerce. Les ports dits de première génération, conçus dans les années 1960, étaient surtout des lieux de
dépôt des marchandises.

Or, l'abaissement des coûts maritimes, par l'aménagement de ces plate-formes portuaires performantes et par l'augmentation de la taille des bateaux de haute mer, exige en amont la diminution des coûts pré et post-acheminement des marchandises.

En el transporte multimodal, los modos de transporte son eslabones de una muy larga cadena que vincula los intereses de los protagonistas del comercio internacional. [...] Por efecto de un vasto y eficiente sistema global de transportes, una red de intereses económicos interactuian alrededor de esas mercaderias que terminarán finalmente vendiéndose en mercados quizás muy distantes. (Fraguio, 1994:2)

Le dynamisme du port rejaillit sur ses zones géographiques d'influence. Elles peuvent s'élargir par maîtrise des coûts de transport terrestre et par mise en concurrence des différents modes (Bourdillon, 1991 : 94). La relation entre le port et les aires qu'il contrôle se resserre, tendant à une véritable "integración de estos puertos con sus respectivos hinterland a través de redes ferroviarias, carreteras e hidrovías eficientes" (La Nueva Provincia, 6 septembre 1996). 
Décidés par les autorités et aménagés en partenariat avec de grands groupes privés, les projets de couloirs de transport multimodaux devraient être réalisés à moyen terme. Ils redéfiniront profondément l'organisation du sytème portuaire brésilien, par une répartition plus judicieuse des expéditions, selon la proximité géographique des zones de production et des points d'expédition. Cependant, leur mise en place sera freinée par des problèmes environnementaux. Dans les documents officiels, préparatoires et prospectifs, et dans les budgets prévisionnels, aucune étude d'impact écologique n'apparaît... Des institutions ont commencé à s'opposer, qui défendent les conditions de vie des populations indiennes localisées près des voies fluviales concernées, et qui insistent sur les conséquences désastreuses de la voie Paraná-Paraguay pour le Pantanal.

RÉSUMÉ : Par la mise en place progressive de couloirs de transport multimodaux, le Brésil modifie sa logistique d'acheminement de ses productions agricoles et réorganise son système portuaire d'expédition des produits issus du complexe soja. La voie fluviale, ouverte sur le Rio Madeira (de Porto Velho au port d'Itacoatiara) et prolongée par la voie maritime de l'Amazone, structure le couloir Nord-Ouest récemment inauguré.

RESUMO : A travês do establecimento progressivo de corredores de transporte multimodais, o Brasil está modificando sụa logística de escoamento de seus produtos agrícolas e está reorganizando seu sistema portuario de exportaçáo de produtos do complexo soja. A hidrovia, aberta no Rio Madeira (de Porto Velho ao porto de Itacoatiara) e prolongada pela via maritima do Rio Amazonas, estrutura o corredor Noroeste recentemente inaugurado. 


\section{Références bibliographiques}

ABIOVE (1996). Données statistiques annuelles sur le complexe soja au Brésil. São Paulo.

BOURDILLON, Jacques (1991). Les réseaux de transport français face à l'Europe. Paris : La Documentation Française. 223 p.

BURNQUIST, Heloisa Lee (coord.) (1994). "O complexo agroindustrial da soja", pp 43-68. In : Liberalização comercial : um fator de desenvolvimento do setor agrícola brasileiro, Brasilia : IPEA, Serie Estudos da Política Agrícola, $\mathrm{n}^{\circ} 14,216 \mathrm{p}$.

Bolsa de Comercio de Rosario (1997). "Improving Brazil's infrastructure". Boletin semanal, Rosario, $n^{\circ} 89,13$ juin, p. 6-8.

CASTRO, Newton de (1995). Intermodalidade, intramodalidade e o transporte de longa distancia no Brasil. Brasília : IPEA, Textos para Discussão, $\mathrm{n}^{\circ} 367$, fevereiro, $21 \mathrm{p}$.

FRAGUIO, Carlos J. (1994). Los puertos a comienzos del siglo XXI. Bahía Blanca : Universidad Nacional del Sur. Ciclo de conferencias. Octubre. $13 \mathrm{p}$.

LICIO, Antonio (1995). "Os eixos estruturadores e os corredores de transportes". Revista de política agricola, out-nov-dez, $\mathrm{n}^{\circ} 4$, ano IV, p. 34

Ministério da Agricultura, do Abastecimento e da Reforma Agrária (MAARA) (1996). A agricultura brasileira : integraçăa sul-americana e entradas/saidas pelo Pacifico. Brasília. Mimeo. 13 p.

Ministério da Agricultura, do Abastecimento e da Reforma Agrária (MAARA) (1996). Corredores de transporte multimodais. Brasilia. Fevereiro. 29 p.

Ministério dos Transportes (1996). Corredor de transporte multimodal noroeste. Brasília. Junho. $36 \mathrm{p}$.

PAVAN, Renato Casali (1994). "Infra-estrutura de transportes", p. 55-60. In : MELLO, Fernando Homem (de) (coord.). Forum Cargill de debates : "O futuro agricola brasileiro". Campinas : Fundaçāo Cargill, 126 p. 
VIEIRA, Luís Fernando (1997). "Complexos agroalimentares do milho, da soja e do trigo no Brasil", p. 115-136. In : Procisur/IICA. Mapeo tecnológico de cadenas agroalimentarias en el Cono Sur. Montevideo, febrero.

- 1997. "Abertura nos portos". Folha de Säo Paulo, 11 mai.

- 1997. "Nova hidrovia no Rio Madeira deve baratear o custo da soja brasileira". Folha de Säo Paulo, 12 avril.

- 1997. "O novo eldorado verde". Veja, 2 avril.

- 1997. "Choveu na horta". Veja, 26 mars.

- 1997. "Amazonas transporta soja do Centro-Oeste". Folha de São Paulo, 25 mars.

- 1997. "Produçăo poderá crescer 4, 5\% no Centro-Oeste". Folha de São Paulo, 15 mars.

-1997. "Maggi é o maior plantador - Novo rei da soja explora fronteiras". Folha de Säo Paulo, 26 janvier.

- 1997. "País perde US $\$ 5$ bi com portos ineficazes". Folha de São Paulo, 6 janvier.

- 1996. "Soja aumenta espaço no cerrado do Piaul". Folha de Säo Paulo, 23 octobre

- 1996. "Particular énfasis en las cargas generales". La Nueva Provincia, Bahia Blanca, 6 septembre.

- 1996. "Esmagadoras pagaráo mais pela soja". Gazeta Mercantil, 5 septembre.

- 1996. "Indústria reduz processamento da soja". Folha de Säo Paulo, 2 septembre.

- 1996. "Corredores tornam a soja mais competitiva no mercado externo". Manchete Rural, São Paulo, avril. 